(D) Check for updates

Cite this: Analyst, 2017, 142, 3356

Received 28th June 2017,

Accepted 18th August 2017

DOI: $10.1039 / c 7 a n 01361 b$

rsc.li/analyst

\title{
Gelatin gels as multi-element calibration standards in LA-ICP-MS bioimaging: fabrication of homogeneous standards and microhomogeneity testing $\dagger$
}

\author{
Martin Šala, * Vid S. Šelih and Johannes T. van Elteren*
}

\begin{abstract}
Highly homogeneous multi-element gelatin calibration standards were fabricated for quantitative LA-ICP-MS bioimaging. Heterogeneity issues caused by the so-called "coffee-stain" and/or "Marangoni" effects were found to be element-dependent but could be circumvented by careful selection of drying/setting conditions. A micro-homogeneity test was developed for certification of the standards.
\end{abstract}

After the first successful attempt of analysis of a biological sample by laser ablation-inductively coupled plasma-mass spectrometry (LA-ICP-MS), ${ }^{1}$ this microanalytical technique has developed rapidly and new $2 \mathrm{D}$ and $3 \mathrm{D}$ bioimaging applications are constantly added..$^{2-5}$ Recent instrumental developments of this widely accepted solid sampling technique ${ }^{6}$ have ultimately led to faster analysis. ${ }^{7,8}$ Because of the high spatial resolution (as low as $1 \mu \mathrm{m}$ for higher elemental concentrations but generally in the range of 5-20 $\mu \mathrm{m})$ and broad dynamic range (10-11 orders of magnitude), the technique is attractive for solving metal biochemistry. ${ }^{9}$ However, the versatility of LA-ICP-MS for $2 \mathrm{D}$ or $3 \mathrm{D}$ bioimaging is hampered by accurate and precise quantification through factors such as sensitivity drift, elemental fractionation, matrix effects, interferences but especially the lack of available matrix matched reference materials. ${ }^{10}$ Non-matrix matched calibration standards need to be combined with single/multiple internal standard normalization to correct for elemental fractionation caused by sensitivity drift, matrix effects as well as the difference in ablation yield between samples and standards. This can be achieved via an additional layer containing the internal standard on or under the samples and calibration standards, ${ }^{11,12}$ by aspirating a known standard solution into the ablation cell or directly in front of the torch, or by using no internal standard but total consumption of a sectioned sample and calibration standard. ${ }^{13}$

Department of Analytical Chemistry, National Institute of Chemistry, Hajdrihova 19, 1000 Ljubljana, Slovenia.E-mail: martin.sala@ki.si, elteren@ki.si

$\dagger$ Electronic supplementary information (ESI) available: Experimental section, Table S1 and Fig. S1-S3. See DOI: 10.1039/c7an01361b
Commonly the quantification relies on in-house prepared calibration standards as described in a recent comprehensive review by Limbeck et $a .^{3}{ }^{3}$ In an often followed protocol by Hare et $a l .{ }^{14}$ standards are tuned to the specific tissue under study by spiking similar homogenized tissue material with the elements of interest followed by cryo-fixing and -sectioning. However, this protocol is laborious, involves handling of biological material and implies that standards must be prepared for each tissue matrix individually. Instead of using spiked matrix-matched tissue resembling the actual matrix of the sample under investigation as closely as possible, another commonly accepted concept is the use of spiked media that mimic the composition of the biological sample. For plant tissues this implies the use of pure cellulose paper, e.g. filter paper, ${ }^{15,16}$ although hydrocolloid gels, e.g. agarose, may be used as well. ${ }^{17}$ For mimicking animal and proteinaceous materials commonly a gelatin gel is chosen, ${ }^{18}$ and sometimes the tissue of interest is mixed with a sol-gel matrix to improve the standard's temporal stability. ${ }^{19}$ These hydrocolloid gel standards have a lot of advantages over the established tissue standards, i.e. simple and fast preparation, no handling of biological material, and the number of analytes and their concentration range can be easily adapted.

For the preparation of multi-element calibration standards we have chosen a gelatin matrix because of its close resemblance to animal tissue and the possibility to fine-tune the required gelatin properties from the type (A or B) and bloom strength (50-300) of commercially available gelatins (difference in gelatin type and blooms strengths can be seen in Fig. S1†). The preparation described in the literature ${ }^{18}$ only works for a limited number of elements, in particular for cations such as $\mathrm{Al}^{3+}, \mathrm{Cr}^{3+}, \mathrm{Fe}^{3+}, \mathrm{Ce}^{3+}, \mathrm{La}^{3+}, \mathrm{Pt}^{2+}$, etc. that exhibit cross-linking of gelatin structures and thus immobilize the element, ${ }^{20}$ resulting in homogeneous standards. However, these limitations have not been noticed before as the focus has mostly been on the use of gelatin as a matrix for calibration standards containing cross-linking cations. ${ }^{18}$ We found that most other elemental anions or cations lead to perceived matrix heterogeneities, either as a result of chromatographic effects upon 
drying, leading to the "coffee stain" effect (visible in the elemental image as higher concentrations at the edges) or as a result of a change in the surface tension gradient caused by a concentration and/or temperature gradient upon drying (visible from higher element concentrations in the center). This incongruent behavior plays a critical role not only in the lateral distribution of elements but also in the depth distribution as will be shown below. Hence the customary preparation procedure needs to be modified to produce a true multielement gelatin calibration standard with high homogeneity. We studied a wide range of parameters that might affect the gel quality and the element distribution, viz. gelatin type (A and B), bloom strength (60-300), concentration of gelatin in (film forming) solution (1-20\% m/v), acidity (0-4\% v/v $\mathrm{HNO}_{3}$ ), element concentration (1-50 $\mathrm{mg} \mathrm{kg}^{-1}$ ) and temperature of drying/setting of the gels $\left(25-120^{\circ} \mathrm{C}\right)$. The $3 \mathrm{D}$ spatial distribution of a variety of elements in the gels was then examined by surface and depth mapping LA-ICP-MS protocols. Detailed fabrication and measurement conditions can be found in the ESI. $\dagger$

The crucial aspect governing the homogeneous distribution of elements during the preparation of gelatin gels is the temperature of drying/setting. This is evident from Fig. 1 in which customary "cold" (room temperature) and optimized "hot" $\left(100{ }^{\circ} \mathrm{C}\right)$ drying/setting procedures are compared for surface and depth distribution of As and Gd in the gels. Although various gel drying/setting temperatures were tested $\left(60-120^{\circ} \mathrm{C}\right)$ in the preparation of $20 \mathrm{mg} \mathrm{kg}^{-1}$ As and $\mathrm{Gd}$ in $10 \% \mathrm{~m} / \mathrm{v}$ gelatin, for clarity only the customary "cold" and optimized "hot" conditions are shown; the lower temperatures still yielded non-optimal distributions and higher than optimal temperatures yielded gelatin films with surface deformations. Fig. 1 depicts surface maps (half of a drop) and depth maps through the center of the drop for Gd and As. It can be seen that drying/setting at room temperature and at $100{ }^{\circ} \mathrm{C}$ yields a homogeneous 3D distribution of Gd in the gelatin drop due to its cross-linking properties, whereas for As the drying/setting temperature was very critical with obvious "Marangoni" effects noticeable at room temperature for both surface and depth distribution but less so at elevated temperatures and practically full 3D As homogeneity was achieved at $100{ }^{\circ} \mathrm{C}$. Other elements show similar behavior and the homogeneity of the standards is increasing with the temperature upon drying/

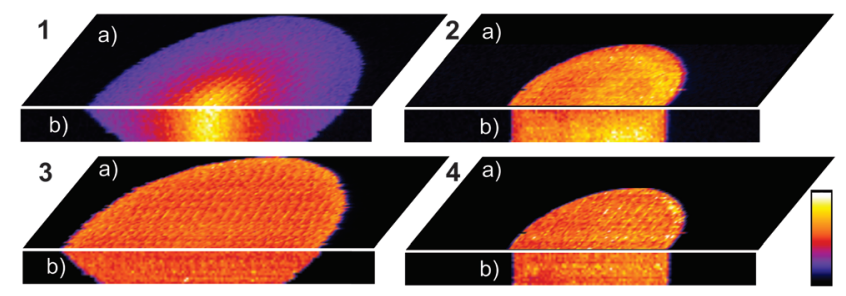

Fig. 1 Elemental distribution of As (1 and 2) and Gd (3 and 4) in gelatins both on the surface (a) and in depth (b) upon drying at room temperature ( 1 and 3$)$ and at $100{ }^{\circ} \mathrm{C}(2$ and 4$)$. setting. It should be noted that drying/setting of the gelatin drop needs to be performed in a mechanical convection oven and not on a hot plate as the latter approach creates the temperature and concentration gradients leading to "Marangoni" effects because of the difference in temperature of the hot plate and the surrounding air.

The effect of acidity on the heterogeneity of multi-element gelatin standards was also studied since elevated acid concentrations may trigger gelatin degradation. ${ }^{20}$ This is demonstrated in Fig. $\mathrm{S} 2 \dagger$ where increasing amounts of nitric acid were added to gelatin solutions showing that concentrations higher than $2 \% \mathrm{v} / \mathrm{v}$ in the preparation phase have a negative effect not only on the $3 \mathrm{D}$ element homogeneity in the gelatin drop but also on the gelatin "film" quality visible as a noneven surface of the film. The gelatin concentration in the starting solution also plays a role in the 3D element homogeneity in the gelatin drop as can be seen from Fig. 2. The depth maps in Fig. 2b, associated with 15 passes on the same line through the center of the drop, show unusual profiles due to slight differences in thickness of the drop with the edge of the drop in all cases several micrometers thicker. As expected the average thickness increases as a function of the initial gelatin concentration, from ca. $0.2 \mu \mathrm{m}$ (at $1 \% \mathrm{~m} / \mathrm{v}$ gelatin) to more than $3 \mu \mathrm{m}$ (at $\geq 10 \% \mathrm{~m} / \mathrm{v}$ gelatin) in the center of the drop.

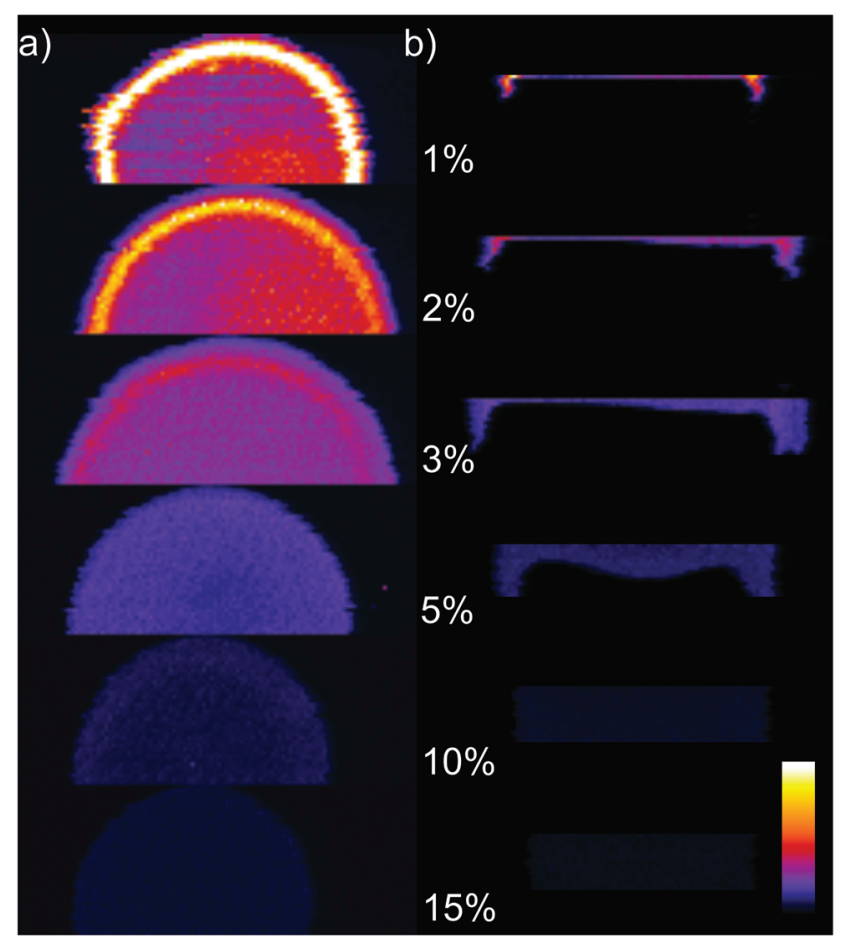

Fig. 2 Distribution of As on the surface (a) and in depth along a line through the center (b) of a dried gelatin drop standard containing different initial contents of gelatin $(1,2,3,5,10$, and $15 \% \mathrm{~m} / \mathrm{v}$; bloom strength 300 , type A). Surface images (a) are scaled correctly in the $x$ and $y$ direction (actual pixel size $=80 \times 80 \mu \mathrm{m}^{2}$ ) whereas depth images (b) are expanded a factor 400 in the $y$ direction (actual pixel size $=80 \times$ $0.2 \mu \mathrm{m}^{2}$ ). 
This implies that the center of the lowest concentration gelatin standard is already penetrated after the first ablation pass whereas the two highest concentration standards are still not penetrated after 15 passes. It is clear that lower initial gelatin concentrations result in worse $3 \mathrm{D}$ element homogeneity, something that can be explained by too fast setting and drying of "diluted" gels, triggering a chromatographic event leading to the "coffee-stain" effect. However, initial gelatin concentrations higher than $15 \% \mathrm{~m} / \mathrm{v}$ are not recommended either as they yield standards that are too viscous to handle comfortably and dry into films that show ripples or bubbles.

Gelatins with the most homogenous element distributions were prepared from $10 \% \mathrm{~m} / \mathrm{v}$ gelatin solutions at $100{ }^{\circ} \mathrm{C}$ and were used for construction of 5- or 6-point calibration graphs; Table $\mathrm{S} 1 \dagger$ summarizes the calibration graph characteristics for 7 elements but from our experience most (anionic or cationic) elements can be used for the preparation of a gelatin standard with a homogeneous element distribution (see also Fig. S3† for a representation in the form of elemental image maps). In all cases the linearity of the calibration graphs expressed as the coefficient of determination $\left(R^{2}\right)$ was better than 0.99 .

To evaluate the microhomogeneity of the prepared gelatin standards a series of experiments with different laser beam sizes (round mask: 5, 8, 10, 12, 15, 20, 25, 35, 40 and $50 \mu \mathrm{m}$ ) and $\mathrm{Cr}$ and As concentrations of $20 \mathrm{mg} \mathrm{kg}^{-1}$ was performed. The average pixel intensity in the drop (retrieved by ImageJ 1.49 software, National Institutes of Health, Bethesda, USA) is given by a value of $A \pm \mathrm{SD}_{\mathrm{t}}$ (in counts) where the standard deviation $\mathrm{SD}_{\mathrm{t}}$ represents the total observed noise in the image due to (i) Poisson-distributed shot noise $\mathrm{SD}_{\mathrm{P}}$ at very low pixel intensities, related to the square root of the pixel intensity $(\sqrt{ } A)$, (ii) Flicker noise $\mathrm{SD}_{\mathrm{F}}$ that is proportional to the pixel intensity $(q \cdot A$, with $q$ a factor between 0 and 1$)$ and (iii) sampling noise $\mathrm{SD}_{\mathrm{S}(\mathrm{D})}$ associated with the element heterogeneity of the gelatin standards and the sampling beam size $D$ (with $D$ the diameter of the round mask or linear dimension along the square mask):

$$
\mathrm{SD}_{\mathrm{t}}^{2}=\mathrm{SD}_{\mathrm{P}}^{2}+\mathrm{SD}_{\mathrm{F}}^{2}+\mathrm{SD}_{\mathrm{S}(\mathrm{D})}{ }^{2}=A+q^{2} \cdot A^{2}+\mathrm{SD}_{\mathrm{S}(\mathrm{D})}{ }^{2}
$$

where $\mathrm{SD}_{\mathrm{S}(\mathrm{D})}$ changes with beam size $D$ according to

$$
\mathrm{SD}_{\mathrm{S}(a \cdot \mathrm{D})}=a \cdot \mathrm{SD}_{\mathrm{S}(\mathrm{D})}
$$

implying that when beam size $D$ increases with a factor $a$, the sampling noise also increases with a factor $a$, thus the relative standard deviation $\operatorname{RSDS}_{(a \cdot \mathrm{D})}$ becomes $100 \times(a \cdot \mathrm{SD}) /\left(a^{2} \cdot A\right)=$ $\mathrm{RSD}_{\mathrm{S}(\mathrm{D})} / a$. The experimental standard deviation data $\mathrm{SD}_{\mathrm{t}}$ for the respective beam sizes were fitted based on the pixel intensity values $A$ using eqn (1) and (2), yielding the unknown factor $q$ (and thus the Flicker noise) and the heterogeneity noise $\mathrm{SD}_{\mathrm{S}(\mathrm{D})}$; the goodness of fit can be observed in Fig. S4. $\dagger$ Fig. 3 summarizes the findings, expressed as $\mathrm{RSD}(=\mathrm{SD} / A)$ values (in \%) $v s$. beam size $D$ (in $\mu \mathrm{m}$ ), for both As and Cr. The homogeneity expressed as $\mathrm{RSD}_{\mathrm{S}(\mathrm{D})}$ is 2.2 and $1.4 \%$ at a beam size $D$ of $5 \mu \mathrm{m}$ for $\mathrm{Cr}$ and As, respectively, making the gelatin standards highly microhomogeneous. Larger beam sizes "smooth"
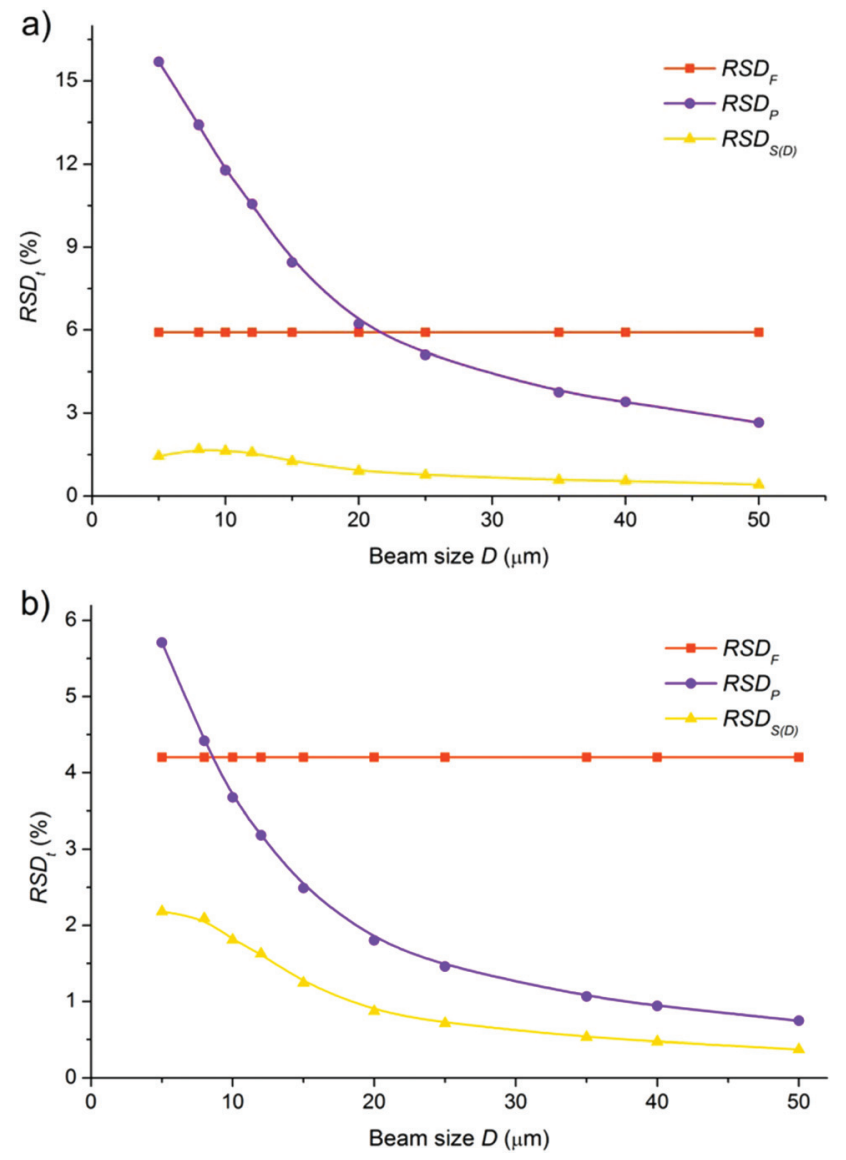

Fig. 3 After fitting the experimental data for total image noise $\mathrm{RSD}_{\mathrm{t}}$ vs. beam size $D$ for the $\mathrm{Cr}(\mathrm{a})$ and As (b) standards (see Fig. $\mathrm{S} 3 \dagger$ ), based on eqn (1) and (2), RSD $\mathrm{t}_{\mathrm{t}}$ could be partitioned among the separate noise contributors such as Flicker noise $\left(R_{S D_{F}}\right)$, Poisson noise $\left(R S D_{P}\right)$ and heterogeneity noise caused by the size of the laser ablation sampling beam $D\left(\operatorname{RSD}_{\mathrm{S}(\mathrm{D})}\right)$.

the signal as evident from $\mathrm{RSD}_{\mathrm{S}(\mathrm{D})}$ values better than $0.5 \%$ at a beam size of $50 \mu \mathrm{m}$. Flicker noise is associated with the ablation process, plasma fluctuation, etc., yielding a constant noise contribution $\mathrm{RSD}_{\mathrm{F}(\mathrm{D})}$ of 4.2 and $5.9 \%$ for $\mathrm{Cr}$ and As, respectively. Poisson noise, related to counting statistics, becomes significant at low pixel intensities $A$ as encountered at low element concentration, short acquisition time or small beam size, the latter variable clearly showing this behavior in Fig. 3. The protocol laid out for microhomogeneity testing is generic in nature and may aid in homogeneity studies of various materials using similar or other imaging techniques.

In conclusion, a procedure for the fabrication of highly homogenous, matrix-matched microanalytical standards for LA-ICP-MS imaging of proteinaceous samples, either of animal or human origin, was developed. Furthermore, a novel microhomogeneity testing protocol showed that the standards fabricated contributed only $c a .2 \%$ of the total image noise at a pixel size of $5 \mu \mathrm{m}$; this protocol is generic in nature and may also be used for testing of other standards and imaging procedures. 


\section{Conflicts of interest}

There are no conflicts to declare.

\section{Acknowledgements}

This research was supported through programme P1-0034 and project N1-0060 by the Slovenian Research Agency.

\section{References}

1 S. Wang, R. Brown and D. J. Gray, Appl. Spectrosc., 1994, 48, 1321-1325.

2 D. J. Hare and E. J. New, Chem. Commun., 2016, 52, 89188934.

3 A. Limbeck, P. Galler, M. Bonta, G. Bauer, W. Nischkauer and F. Vanhaecke, Anal. Bioanal. Chem., 2015, 407, 65936617.

4 D. J. Hare, E. J. New, M. D. de Jonge and G. McColl, Chem. Soc. Rev., 2015, 44, 5941-5958.

5 J. S. Becker, A. Matusch and B. Wu, Anal. Chim. Acta, 2014, 835, 1-18.

6 D. Gunther and B. Hattendorf, TrAC, Trends Anal. Chem., 2005, 24, 255-265.

7 A. Gundlach-Graham, M. Burger, S. Allner, G. Schwarz, H. A. O. Wang, L. Gyr, D. Grolimund, B. Hattendorf and D. Gunther, Anal. Chem., 2015, 87, 8250-8258.
8 S. J. M. Van Malderen, A. J. Managh, B. L. Sharp and F. Vanhaecke, J. Anal. At. Spectrom., 2016, 31, 423-439.

9 M. Opačić, A. J. Ristić, D. Savić, V. S. Šelih, M. Živin, D. Sokić, S. Raičević, V. Baščarević and I. Spasojević, Metallomics, 2017, 9, 141-148.

10 M. Burn, P. Lanari, T. Pettke and M. Engi, J. Anal. At. Spectrom., 2017, 32, 1359-1377.

11 C. Austin, D. Hare, T. Rawling, A. M. McDonagh and P. Doble, J. Anal. At. Spectrom., 2010, 25, 722-725.

12 M. Bonta, H. Lohninger, M. Marchetti-Deschmann and A. Limbeck, Analyst, 2014, 139, 1521-1531.

13 D. A. Frick, C. Giesen, T. Hemmerle, B. Bodenmiller and D. Gunther, J. Anal. At. Spectrom., 2015, 30, 254-259.

14 D. J. Hare, J. Lear, D. Bishop, A. Beavis and P. A. Doble, Anal. Methods, 2013, 5, 1915-1921.

15 M. A. G. Nunes, M. Voss, G. Corazza, E. M. M. Flores and V. L. Dressler, Anal. Chim. Acta, 2016, 905, 51-57.

16 M. Bonta, B. Hegedus and A. Limbeck, Anal. Chim. Acta, 2016, 908, 54-62.

17 H. J. Stärk and R. Wennrich, Anal. Bioanal. Chem., 2011, 399, 2211-2217.

18 D. Gholap, J. Verhulst, W. Ceelen and F. Vanhaecke, Anal. Bioanal. Chem., 2012, 402, 2121-2129.

19 H. Sela, Z. Karpas, H. Cohen, Y. Zakon and Y. Zeiri, Int. J. Mass Spectrom., 2011, 307, 142-148.

20 R. Schrieber and H. Gareis, Gelatine Handbook: Theory and Industrial Practice, WILEY-VCH Verlag GmbH \& Co., Weinheim, 2007. 